REVISTAS DE LA FAHCE

Faculad de Hunuridads y Cenoirs de la Eduacoion | UMU?
Educación Física y Ciencia

ISSN: 1514-0105

ISSN: 2314-2561

revistaefyc@fahce.unlp.edu.ar

Universidad Nacional de La Plata

Argentina

\title{
Educação Física e promoção da saúde: uma revisão de perspectivas teórico- metodológicas no Brasil
}

Antunes, Diogo S. H; Knuth, Alan G.; Damico, José Geraldo

Educação Física e promoção da saúde: uma revisão de perspectivas teórico-metodológicas no Brasil

Educación Física y Ciencia, vol. 22, núm. 1, 2020

Universidad Nacional de La Plata, Argentina

Disponible en: http://www.redalyc.org/articulo.oa?id=439963095008

DOI: https://doi.org/10.24215/23142561e116

Esta obra está bajo una Licencia Creative Commons Atribución-NoComercial-Compartirlgual 4.0 Internacional. 


\title{
Educação Física e promoção da saúde: uma revisão de perspectivas teórico- metodológicas no Brasil
}

\author{
Physical Education and health promotion: a review of theoretical-methodological perspectives in Brazil
}

Diogo S. H Antunes

Escola Amigos do Verde / Escola Lupicinio Rodrigues, Brasil

diogoyantunes@gmail.com

DOI: https://doi.org/10.24215/23142561e116

Redalyc: http://www.redalyc.org/articulo.oa? id $=439963095008$

Alan G. Knuth

Universidade Federal do Rio Grande / Universidade

Federal de Pelotas, Brasil

alan_knuth@yahoo.com.br

José Geraldo Damico

Universidade Federal do Rio Grande do Sul /

Universidades Federal do Rio Grande / Universidade

Federal de Santa Maria, Brasil

jgdamico@gmail.com

Recepción: 03 Agosto 2018

Aprobación: 14 Diciembre 2019

\section{Resumo:}

Na complexidade da atuação da educação física na promoção da saúde é possível identificar diferentes perspectivas epistemológicas no saber-fazer dos trabalhadores. A intenção deste artigo, de caráter ensaístico, é apontar estas perspectivas, buscando perceber suas influencias teóricas e históricas, assim como os caminhos que vem percorrendo em publicações, autores que se destacam e focos de interesse. Por meio de buscas não sistemáticas em bancos de dados e periódicos, foram encontradas publicações e marcos legais que ligam educação física e promoção da saúde. Conclui-se a existência de quatro perspectivas teórico-metodológicas: epidemiológica, aponta a relação positiva entre a atividade física e a menor incidência de doenças; pós estruturalista ligada a produção de subjetividade e relações sociais, na promoção da saúde; materialismo histórico-dialético reflete a respeito das inter-relações sociais e dos meios de produção e exploração; e a perspectiva integrativa ligada a uma perspectiva vitalista, onde a saúde passa a ser percebida como a busca da inteireza do ser. É importante ressaltar que a apresentação destas perspectivas de forma linear é um recurso didático, mas que na realidade da prática profissional nem sempre mostram-se com fronteiras claramente delineadas, podendo se apresentar de forma hibrida e inter-relacionada.

PalaVras-chave: Promoção da saúde, Perspectivas teórico-metodológicas, Educação física.

\section{ABSTRACT:}

In the complexity of the performance of Physical Education in health promotion, it is possible to identify different epistemological perspectives in the know-how of workers. The aim of this article, of an essay character, is to point out these perspectives, seeking to perceive its theoretical and historical influences, as well as the paths it has been going through in publications, authors that stand out and focuses of interest. Through non-systematic searches in databases and journals, publications and legal frameworks linking Physical Education and health promotion were studied. The existence of four theoretical-methodological perspectives was found: epidemiological, which points out the positive relation between physical activity and the lower incidence of diseases; post structuralist, which links to the production of subjectivity and social relations in the promotion of health; historical-dialectical materialism, which reflects on the social interrelationships and the means of production and exploitation; and the integrative perspective, which links to a vitalist perspective, where health comes to be perceived as the pursuit of the wholeness of being. It is important to emphasize that the presentation of these perspectives in a linear way is a didactic resource, but that in the reality of the professional practice they do not always show themselves with boundaries clearly defined, being able to present themselves in a hybrid and interrelated way.

KEYwORDS: Health promotion, Theoretical-methodological perspectives, Physical education. 


\section{INTRODUÇÃO}

A educação física é uma área dinâmica, com atuação nos campos da educação, saúde, esporte e lazer. Além da inserção no contexto privado do campo da saúde, a recente inserção na saúde coletiva, em especial a partir da atuação no Sistema Único de Saúde (SUS), tem fortalecido esta área de atuação e apresentado um cenário complexo e contraditório, que manifesta-se a partir de diferentes linhas teórico metodológicas que coexistem no saber-fazer da prática da educação física. Neste trabalho, buscamos mapear as perspectivas teórico-metodológicas ligadas ao núcleo da educação física e saúde e mais especificamente promoção da saúde, apresentando autores, publicações e focos de interesse que se destacam em cada uma das perspectivas observadas. A compilação de tais perspectivas com recorte para o campo da promoção saúde é propositalmente arbitrária. Não há interesse em estabelecer um formulário definitivo. É legítimo considerar que as perspectivas e autores aqui localizados poderiam ser diferentemente modelados se a pesquisa assumisse outros caminhos e critérios

Alguns marcos legais ajudam a delimitar o que estamos considerando Promoção da Saúde, não com uma proposição conceitual, e sim com uma narrativa de como esta área foi sendo produzida amparada nestes termos. Assim, a resolução 218/97 do Conselho Nacional de Saúde reconhece a educação física como uma das áreas profissionais da saúde (CNS, 1997), a Política Nacional de Promoção da Saúde traz as práticas corporais/ atividade física como uma das ações específicas a serem implementadas no SUS (Brasil, 2006a) somados a efetivação dos Núcleos de Apoio à Saúde da Família (NASF) dos Centros de Atenção Psicosocial (CAPS) do programa Academia da Saúde, Consultório na rua (CnR) e Unidades de Acolhimento ofereceram as bases políticas de alocação de um fazer profissional na complexidade do contexto que envolve o campo da saúde coletiva, e consequentemente tencionam o aprofundamento de algumas reflexões a respeito da educação física e promoção da saúde nestas inter-relações.

Este panorama se coloca em um momento histórico em que a saúde tem sofrido reduções a um cuidado direcionado para os riscos, e entre eles o movimento humano vem recebendo atenção e olhares acadêmicocientíficos, em função de sua relação com a morbimortalidade da população, especialmente o modelo vigente bastante localizado nas condições crônico-degenerativas. Tais elementos nos instigaram a refletir a respeito das transformações paradigmáticas que vem ocorrendo no campo da educação física em suas inter-relações com o campo da saúde (Campos, 2000), influenciados por transformações maiores que tem ocorrido na ciência contemporânea.

Dois trabalhos publicados recentemente podem contextualizar o interesse, e ao mesmo tempo, a divergência paradigmática sobre os olhares para a saúde que circundam a educação física. Ramires et al. (2014) e Neves, Antunes, Baptista y Assumpção (2015) revisam trabalhos acadêmicos sobre epidemiologia da atividade física e educação física no campo da saúde pública, respectivamente. Apesar de não localizarem perspectivas teórico-metodológicas presentes nestes cenários ambos narram um aumento abrupto de produções e interesse pelo campo da saúde. Neves et al. (2015) localizaram 60 trabalhos entre 2000 e 2012 com características que situam a educação física na saúde pública. O trabalho de Ramires et al. (2014) é mais específico para a epidemiologia da atividade física, ainda assim, entre 2005 e 2013 um total de 276 artigos foram identificados. Foi relatado um incremento de 7 para 49 artigos por ano nesta temática que se situa na saúde. Os critérios das pesquisas, as diferenças e afastamentos das perspectivas faz com que não necessariamente os mesmos estudos tenham sido incluídos e analisados pelos autores e mesmo assim são trabalhos que orbitam pelo interesse da educação física filiada à saúde.

No campo da saúde temos tradicionalmente as questões relativas à atenção, promoção, prevenção, tratamento e reabilitação. O foco deste trabalho é mapear analiticamente as perspectivas teóricometodológicas que circundam o saber-fazer de trabalhadores de educação física no campo da promoção da saúde no Brasil, tendo como base produções acadêmico-científicas e marcos legais. Também fará parte desta discussão um apanhado histórico buscando descrever brevemente as origens destas perspectivas, buscando 
estabelecer conexões e afastamentos em seus modos de saber-fazer, contribuindo com o cenário teóricoprático da educação física em associação à promoção da saúde.

Este artigo assume um estilo ensaístico. Foram realizadas buscas não sistemáticas em bancos de dados buscando trabalhos brasileiros. As buscas não visaram esgotar as publicações na área, mas identificar as perspectivas teórico-metodológicas presentes nas publicações e marcos legais relacionados à educação física e promoção da saúde. Para tanto é possível observar atualmente diferentes perspectivas teórico-metodológicas que convivem simultaneamente na prática da educação física. Mais do que a busca da certeza de respostas que marcam limites e trazem estagnação, temos a intenção de trazer problematizações e reflexóes que instigam e mantém o debate em constante movimento. Puderam ser identificadas quatro perspectivas ligadas à lógica de promoção da saúde na área da educação física, a saber, epidemiológica, pós estruturalista, materialismo bistórico-dialético e integrativa.

$\mathrm{Na}$ contemporaneidade, o dualismo entre atividades físicas e práticas corporais explorado por Damico e Knuth (2015), pode representar em parte a demarcação do que entendemos como condição mínima para o presente texto. Não vemos como possível isolar-se em uma perspectiva teórica sem dialogar ou receber atravessamentos de outras. Filiar-se cegamente a uma tendência acaba por capturar e sedimentar, sem prestigiar a ampla e complexa rede de conceitos e entendimentos que cerca a educação física na promoção da saúde. Os referidos autores, por exemplo, avaliam que uma fuga do objeto (atividades físicas e/ou práticas corporais) para buscar sentido no encontro intercessor com os usuários no cotidiano dos serviços de saúde se apresenta como uma possibilidade de potencializar uma lógica usuário-centrada no SUS. Este é apenas um dos embates pertinentes ao cenário da educação física e saúde. A sequência do presente trabalho pretende explorar as perspectivas teórico-metodológicas identificadas na lógica de promoção da saúde, sua constituição histórica, conexões e afastamentos.

\section{Metodologia}

Este artigo é uma revisão da literatura que assume um estilo ensaístico. Foram realizadas buscas não sistemáticas nos bancos de dados dos Periódicos CAPES, Scielo, Portal de pesquisa da biblioteca virtual em Saúde, biblioteca digital brasileira de teses e dissertações, Google Acadêmico e Google para trabalhos brasileiros, que incluíram artigos, teses, dissertações, livros e marcos legais acerca da temática. As buscas não visaram esgotar as publicações na área e foram realizadas até o momento em que as análises passaram a mostrar saturação, com repetições a cerca dos achados. A metodologia utilizada possibilitou identificar quatro perspectivas teórico-metodológicas presentes nas publicações e marcos legais relacionados à educação física e promoção da saúde.

\section{Breves reflexões sobre paradigmas e eduCAÇÃo física}

Um Paradigma é um sistema de crenças, modelos, representações e interpretações de mundo reconhecidas dentro de um determinado grupo que fornecem problemas e soluções modelares para uma comunidade científica (Kuhn, 1992). Em outras palavras, são crenças que formam o alicerce de uma determinada forma de pensar-agir que embasa todas as demais verdades que sobre ela são construídas. Desta forma, a compreensão de o que é o ser humano, como se desenvolve sua dinâmica de vida, o que é o processo saúde-doença, os sistemas de diagnóstico e tratamento, o que é doença e como preveni-las, está alicerçado sobre determinado paradigma.

Cabe argumentar, que nossa intenção não é realizar um exercício de engavetamento de certas tendências da educação física na sua inserção no campo da promoção da saúde como se determinados autores formassem 
um todo homogêneo sem fissuras, disputas ou efeitos de verdade dissonantes. O artigo de Mathias Loch fornece uma pista nesta direção:

A “Atividade Física e Saúde”, enquanto uma área/sub- -área/linha de pesquisa, parece assumir um significado bastante genérico, sendo praticamente um conceito "guarda chuva”, onde quase tudo cabe, desde estudos que investigam efeito de programas formais e bem controlados de exercícios físicos sobre desfechos biológicos (com humanos e com animais), até estudos sobre a influência de determinantes sociais, culturais e ambientais sobre a prática ou não de atividades físicas. Ou seja, se por um lado, existem estudiosos da "Atividade Física e Saúde” preocupados essencialmente com aspectos biológicos, outros irão fundamentalmente buscar o entendimento de questões mais amplas ligadas a pratica de atividade física. Muitas vezes esta diferenciação passa despercebida pelos críticos, que na maioria das vezes rapidamente rotulam todas as produções dessa área como sendo oriundas das ciências biológicas (Loch, 2012, p.48)

Loch (2012) ao analisar os programas de pós-graduação Stricto Sensu, relacionados à “Atividade Física e Saúde", constata uma heterogeneidade da descrição das áreas de concentração ou linhas de pesquisa, concluindo que existe na "Atividade Física e Saúde" uma interessante possibilidade de convivência entre diferentes olhares.

Na medida em que diferentes Paradigmas que impactam a educação física se transformam, se transformam também todas as questões que se encontram dentro desta área, como por exemplo, a concepção de sujeito, de saúde, de potencialidade de atuação com as práticas corporais/atividades físicas, etc. É importante salientar que a emergência de novos paradigmas não aniquila a perspectiva anterior, em geral, elas seguem coexistindo, com maior ou menor força de uma ou de outra(s), mostrando-se também de forma hibrida e inter-relacionada nas práticas dos sujeitos e coletivos.

Como comentamos anteriormente, observamos que são quatro as principais perspectivas teóricometodológicas da educação física que se articulam ao campo da saúde, por meio da noção de promoção da saúde no Brasil atualmente, a abordagem epidemiológica, a pós-estruturalista, o materialismo histórico-dialético e a ainda emergente perspectiva integrativa.

\section{ABORDAGEM EPIDEMIOLÓGICA DA ATIVIDADE FÍSICA E SAÚDE}

Os estudos que localizam a prática de atividade física no campo da saúde, com delineamentos epidemiológicos de fundo, tem uma primeira influência histórica a partir dos estudos de Morris na década de 50 do século passado, onde foi possível apontar relação positiva entre a atividade física realizada no trabalho e a menor incidência de doenças cardiovasculares (Florindo \& Hallal, 2011). A própria definição de atividade física encontra em Caspersen, Powel e Christenson (1985) um consenso importante para a área e os estudos que aí se localizam passam a operar com este conceito, onde diferentes domínios de atividade física são descritos: trabalho, lazer, deslocamento e ambiente doméstico. A premissa fundamental da aproximação desta abordagem com a saúde está na prevenção e recuperação que a atividade física proporcionaria aos casos de doenças cardiovasculares e posteriormente a um conjunto maior de doenças crônico-degenerativas, o que se relacionava com os padrões de transição nutricional, demográfica e epidemiológica que o Brasil passava. Nessa linha, tais efeitos, além de um acúmulo de evidências científicas, passaram a ser incorporados em políticas públicas, especialmente as de saúde, com interesse prevencionista, de reabilitação, mas especialmente revestido com o discurso de promoção da saúde. Ainda que nem mesmo a noção de promoção da saúde possa ser compreendida com apenas uma área do conhecimento ou política, é contundente nesta abordagem a utilização da noção de promoção da saúde como apoio discursivo e de sustentação. Tanto que no auge desta abordagem nasce também a PNPS brasileira, com influência destes atores e abordagem. Posteriormente é criado também o Programa Academia da Saúde que tem íntima relação com a recente PNPS.

Ainda que assumam conexões íntimas, a abordagem com apoio nos estudos epidemiológicos da atividade física, não pode ser tomada como idêntica à da aptidão física relacionada à saúde, que teve lugar central entre os anos 80 e 90, especialmente na educação física escolar. Há diferenças conceituais, de aplicação 
profissional, de aproximação com o surgimento do SUS e dos delineamentos metodológicos que sustentam as pesquisas. Sobre as diferenças entre as duas formas de pensar a saúde no âmbito escolar sugerimos o texto de Knuth e Loch (2014). Uma das ferramentas que diferenciam a "aptidão física" da "atividade física" é a atenuação do discurso enfático do exercício físico para uma branda mensagem de atividade física, onde se assumiu que o acúmulo de atividades físicas ao longo dos dias, mesmo que em sessões curtas, poderia trazer consequências interessantes para a saúde. Anteriormente não se aceitava tal flexibilização no discurso. Foi a partir das evidências epidemiológicas que se abrandou o discurso e que as recomendaçóes passaram a enfatizar mais o processo atividade física e menos o produto aptidão física. Nesse cenário a atividade física conversou mais diretamente com a lógica de promoção da saúde, se apoiou nos estudos epidemiológicos, especialmente aqueles de delineamentos transversal e coorte e teve ligações viscerais com as políticas públicas de saúde. Portanto, neste panorama os estudos tem priorizado o diagnóstico populacional sobre a prática de atividade física e comportamento sedentário e os aspectos que determinam ou condicionam tais práticas em diferentes grupos populacionais. Há, indubitavelmente, uma ampliação das investigações antes puramente isoladas em variáveis biológicas para variáveis do ambiente físico e social. Também emergem dessa perspectiva as evidências que apostam nos programas comunitários de promoção da atividade física como ferramenta de prevenção e promoção da saúde e que reforçam o discurso Estatal, deslocando recursos e esforços para que as cidades induzam a contratação de trabalhadores da educação física para ministrar aulas à comunidade, reforçando a pauta de incremento dos níveis populacionais de atividade física.

\section{Abordagem pós-estruturalista}

Essa abordagem produz um deslocamento a partir do que se convencionou chamar de teorias Pós-Críticas ao descentrar a ênfase nas dinâmicas de classe e consolidar a importância dos processos de produção de subjetividade, particularmente expressos nas construções discursivas para a elaboração de análises sociais.

Utilizar o referencial pós-estruturalista significa, em primeiro lugar, questionar o que é a realidade, quem são os indivíduos e como estes se relacionam socialmente para promover saúde. Esta perspectiva teórica considera que a realidade e as verdades de cada tempo são construções sociais produzidas na tensão entre os discursos dominantes e os discursos emergentes, que procuram manter ou modificar certos entendimentos e práticas sociais estabelecidas. Assim, as noções de progresso, racionalidade e verdade que são próprias do ideário modernista, no qual o paradigma crítico-social se insere, passam a ser vistas como parte do discurso dominante do final do século XX, um discurso possível entre outros.

O pós-estruturalismo compreende igualmente que no momento em que as pessoas são constituídas dos mesmos discursos que compõem o seu "exterior", torna-se difícil distinguir interioridade de exterioridade, colocando em questão a noção de sujeito autônomo, com um self independente e com possibilidade de livre escolha.

É no campo do pensamento pós-estruturalista que as contribuições do filósofo francês Michel Foucault permitem aprofundar a compreensão sobre o ideário de Promoção à Saúde e, nele, da temática do poder e da produção de subjetividade. Ao opor-se à concepção negativa de poder da tradição política e filosófica moderna, que identifica poder com o Estado e tende a confundir relações de poder com relações de dominação, Foucault preconiza a existência de uma concepção positiva afirmando que

\footnotetext{
Quando se fala de poder, as pessoas pensam imediatamente em uma estrutura política, em um governo, em uma classe social dominante, no senhor diante do escravo, etc. Não é absolutamente o que penso quando falo das relações de poder. Quero dizer que nas relações humanas, quaisquer que sejam elas - quer se trate de comunicar verbalmente [...] ou se trate de relações amorosas, institucionais ou econômicas -, o poder está sempre presente: quero dizer, a relação em que cada um procura dirigir a conduta do outro. São, portanto, relações que se podem encontrar em diferentes níveis, sob diferentes formas; essas relações de poder são móveis, ou seja, podem se modificar, não são dadas de uma vez por todas (Foucault, 2004, p.270)
} 
Esta analítica de poder foucaultiano permite compreender, com maior propriedade, estratégias e mecanismos que são utilizados para governar indivíduos e o social. É possível entender, por exemplo, que as dimensões criativas e instituintes do poder podem gerar, simultaneamente, formas de docilização e de resistência/criação. Esta formulação paradoxal - que desafia a necessidade que temos de atribuir valor a tudo para orientar nossa compreensão do mundo - amplia, em nossa opinião, as possibilidades críticas no que se refere às relações de poder no campo da saúde e a algumas das estratégias centrais ao movimento da Promoção à Saúde.

Na perspectiva pós-estruturalista, o corpo, objeto de saber da Promoção à Saúde, assume uma dimensão que vai além do biológico, constituindo um território de encontro do indivíduo e do coletivo e é, igualmente, significado por dimensões sociais, econômicas, culturais e políticas de um determinado período histórico. Ocorre, aqui, um apagamento das divisões entre o micro e o macro e a constituição de um espaço social que é simultaneamente híbrido e próprio que faz olhar sobre temas e estratégias recorrentes ao projeto de Promoção à Saúde. Estratégias, aparentemente inocentes e naturais, como a intervenção sobre a dieta dos indivíduos objetivando a prevenção e (ou) a redução da obesidade, podem contribuir para a saúde das pessoas e, paradoxalmente, constituir práticas de controle sobre os indivíduos e coletivos.

Entre algumas das publicações que se orientam a partir desta perspectiva está o livro Educação Física e saúde coletiva (Fraga \& Wachs, 2007) e os três volumes intitulados saúde em debate na educação física (Bagrichevsky, Estevão \& Palma, 2003, 2006, 2007). Nas discussões destas obras encontram-se diversos temas tais como a formação profissional, educação física escolar, interfaces com saúde mental e saúde da família, qualidade de vida, desigualdades sociais, políticas públicas em saúde, estilo de vida, imagens de corpo, apenas para citar alguns exemplos que nos permitam perceber a diversidades de temáticas abordadas nas interfaces entre educação física e saúde orientadas pela abordagem pós estruturalista.

\section{MATERIALISMO HISTÓRICO-DIALÉTICo}

Influenciado pelos ideais do materialismo dialético, perspectiva desenvolvida por Karl Marx e Friedrich Engels, esta perspectiva traz como principais contribuições para o campo da educação física as reflexões a respeito da prática da educação física e as inter-relações sociais e dos meios de produção e exploração. Trazem a construção de perspectivas emancipatórias, e libertárias, centradas em uma abordagem crítico superadora, desenvolvendo reflexões sobre valores como solidariedade, cooperação, distribuição, liberdade de expressão dos movimentos ao invés do individualismo da disputa, da apropriação, negando a dominação do homem pelo homem (Coletivo de Autores, 1992).

Esta perspectiva que influenciou intensamente a educação física em seus debates e prática no campo da educação escolar parece ter hoje pouca presença no campo da promoção da saúde, mas são encontradas algumas publicações em especial de Tadeu Baptista. O autor supra citado, discute a promoção da saúde, inferindo que uma vez que esta está relacionada a aspectos amplos, relacionados as condições de vida dos sujeitos, então ha a necessidade de se ponderar sobre a ideia de políticas públicas mais amplas (que extrapolem a questão das práticas corporais) para o desenvolvimento do processo de promoção da saúde. Ainda Infere a respeito da:

incapacidade de os conceitos de atividade física e exercício conseguirem atender as demandas de uma sociedade autônoma e emancipada. A consideração meramente biológica destes conceitos esvazia e desrespeita a dimensão cultural e, portanto, a inserção do corpo nas condições de trabalho, entendido aqui como a relação entre homem e natureza (Baptista, 2013).

O autor em conjunto com Anita Resende debate ainda a relação da manutenção da saúde do trabalhador como forma de reduzir a ausência nos postos de trabalho e com isso, aumentar a certeza da capacidade produtiva do trabalhador (Baptista \& Resende, 2009). Baptista (2013) aposta na compreensão das práticas corporais como proposta que possibilita a aproximação do ser humano consigo, condição sem a qual, ele 
não alcança a integridade necessária ao rompimento da alienação e reificação, por serem elas uma causa fundamental no desenvolvimento de uma série de agravos à saúde humana.

Em outra obra do mesmo autor (Baptista, 2007) este discute como a forma do corpo é determinada pelos interesses relacionados à produção e ao consumo vigentes no modo de produção capitalista. Coloca em debate a manutenção da saúde do trabalhador como forma de reduzir a ausência nos postos de trabalho e, com isso, aumentar a certeza da capacidade produtiva do trabalhador e de que forma a utilização das práticas corporais se manifesta nesta intersecção "manutenção da saúde x manutenção da capacidade produtiva”.

\section{Perspectiva integrativa}

A perspectiva integrativa ampara-se sobre os paradigmas emergentes, que são desenvolvidos a partir da descoberta da física quântica por Niels Bohr e Werner Heisenberg no início do século XX. A mecânica quântica gera uma ruptura com a Física Newtoniana, uma das bases do paradigma Moderno (Dalla Zen, 2010; Goswami, Reed \& Goswami, 2008; Nicolescu, 2005). Posteriormente, têm sido fortalecidos por descobertas de outros campos da ciência, em especial da psicologia Transpessoal e da biologia (Radin, 2008; Sheldrake, 1993; Grof, 2000).

Nas perspectivas emergentes a saúde passa a ser percebida como a busca da inteireza do ser, intimamente ligado com uma concepção vitalista, o que possibilita um repensar das práticas e caminhos para promoção da saúde (Pozatti, 2007; Luz, 2007). A principal manifestação desta perspectiva teórico-metodológica são as práticas corporais integrativas (Yoga, Tai Chi, Terapias corporais, etc.), das quais cabe realizarmos alguns apontamentos. O primeiro, é que há uma vasta produção de textos com relação a estas práticas já produzida. Estas publicações de maneira geral foram feitas por Mestres e professores e não partiram das atuais discussões a respeito da educação física e promoção da saúde, apesar de muito relevantes entendemos que estão fora do escopo deste trabalho.

O segundo é que a terminologia utilizada para denominar as práticas corporais nesta perspectiva ainda encontra divergências, sendo que alguns estudos tratam como praticas corporais alternativas, outros como praticas corporais integrativas, termo que adotaremos neste artigo.

"O termo alternativo é resultante do movimento da contracultura, ocorrido na década de 60, e referese a uma maneira de pensar e agir fora dos padrões da modernidade ocidental” (Coldebella, 2002). A passagem atual para o termo, praticas corporais integrativas, vem conjuntamente com a discussão das Praticas Integrativas e Complementares e com uma integração destas com os avanços epistemológicos dos paradigmas emergentes.

Quatro elementos nos apontam a evidência da existência da perspectiva integrativa na educação física ligada a promoção da saúde. São eles: A atuação profissional com práticas corporais integrativas, marcos legais relacionados a esta perspectiva teórico-metodológica, publicações científicas e disciplinas de graduação e atividades de extensão oferecidas em universidades tendo como temática as práticas corporais integrativas. Sobre este último aspecto, temos como exemplo a universidade federal do Ceará, Itajubá, Alagoas e Rio Grande (UFC, 2015; UFAL, 2015; UNIFEI, 2015; Antunes, 2014)

Um dos marcos atuais mais importantes da influência dos paradigmas emergentes no que se relaciona a promoção da saúde no Brasil é a Política Nacional de Práticas Integrativas e Complementares (Brasil, 2006b). A criação desta política tem incentivado e fortalecido a inserção de Práticas Corporais Integrativas no SUS, e tem fomentado publicações científicas a respeito desta temática.

As publicações relacionadas às Práticas Corporais Integrativas e promoção da saúde são ainda em número pequeno. Encontramos algumas publicações que debatem a importância destas práticas para promoção da saúde (Santos \& Tesser, 2012; Mizuno \& Monteiro, 2012; Frosi \& Pozatti, 2011; Luz, 2007; Matthiesen, 1999), outras que relatam o desenvolvimento de experiências e seus resultados (Fiocruz, 2015; Baptista et al., 2014; Corrêa, et. al. 2014; Antunes, 2011 e 2014; Cesana, 2011; Frosi, 2010; Barollo \& Cabral, 2012). 
Outro ponto relevante encontrado é a existência de ações relacionadas às práticas corporais integrativas no SUS (Santos, 2009; São Paulo, 2012; Brasilía, 2012; Brasil, 2014; Antunes, 2014). É importante apontar que:

O Brasil difere de outros países na oferta de práticas integrativas e complementares, pois predominam as práticas corporais, principalmente nos serviços públicos, o que não coincide com os estudos realizados em países da América Latina (Sousa, Bodstein, Tesser, Santos \& Hortale, 2012)

A concepção integrativa na Educação Física e promoção da saúde é uma perspectiva teórico-metodológica ainda pouco explorada em especial pelas publicações científicas, mas que vem fortalecendo-se nos últimos anos e já é uma realidade no campo da promoção da saúde tanto a nível público a través dos serviços do SUS, como privado, em escolas tradicionais que há décadas desenvolvem atividades relacionadas a estas práticas.

\section{Conchusões}

A educação física é um núcleo profissional amplo, complexo, de distintas interfaces e campos de atuação. Em suas interconexões com a promoção da saúde, conclui-se da existência de quatro perspectivas teórico metodológicas. Apesar de a metodologia utilizada ter sido uma revisão de literatura não sistemática, é possível observar uma maior influência no conteúdo dos marcos legais e número de publicações, ligados às perspectivas epidemiológica e pós estruturalista, ao passo em que a perspectiva integrativa é uma abordagem ainda incipiente e o materialismo histórico dialético, que nas décadas passadas influenciou fortemente as interfaces da educação física com a educação, encontra-se presentes nas publicações de apenas alguns autores.

Acreditamos ser importante que o trabalhador da educação física atuante no campo da saúde tenha conhecimento e clareza a respeito das múltiplas perspectivas teórico-metodológicas que influenciam os debates e práticas nesta área. A complexidade dos desafios sociais, culturais, ecológicos e econômicos que enfrentam as comunidades em um contexto globalizado, tencionam a necessidade de visões claras, abrangentes e sistêmicas, para que sejamos capazes de responder adequadamente a estas questões.

Ao nosso conhecimento esta é a primeira pesquisa a evidenciar perspectivas teórico-metodológicas atuantes na educação física e promoção da saúde. Outros trabalhos têm se debruçado a revisar publicações de forma genérica como o de Neves et al. (2015) ou de uma área específica da saúde como o de Ramires et al. (2014). Há disponível na literatura outras revisões, sejam sistemáticas ou narrativas sobre a recente e maior aproximação da educação física junto às políticas públicas de saúde, aos conceitos de prevenção e promoção da saúde ou a determinados recortes específicos como os trabalhos de Damico e Knuth (2015) e Loch (2012). Todas estas contribuições não se propõem a avançar na leitura das prováveis perspectivas, aqui apresentadas.

Como já exposto, não há uma intenção de categorizar de maneira estática os autores em perspectivas teórico-metodológicas. Mesmo que haja diferenças significativas entre estas perspectivas, que nos possibilitem um debate teórico a respeito de suas histórias, características e bases conceituais, muitas vezes, na realidade experienciada do mundo vivido, não há fronteiras claras que delimitem separações. Em seus encontros forma-se um continuum onde há hibridismos, aproximações, confluências e conflitos. A forma como aqui apresentamos foi uma alternativa textual e explicativa sobre os enquadramentos conceituais em que tais autores foram se localizando. Certamente não se limita apenas aos referenciais apontados, ainda que manifeste uma sumarização fiel ao que pudemos localizar na literatura visitada.

Reconhecemos que estas distintas perspectivas teórico-metodológicas observam "a mesma” realidade de distintos pontos de vista. Elas surgiram em diferentes momentos históricos com a contribuição de diversos autores. Sem dúvida não é interessante que uma ou outra perspectiva domine e defina a operacionalidade da profissão no setor saúde, e esta é uma característica salutar do núcleo da educação física, uma vez que é fundamental reconhecer que na atuação em saúde o ponto central da prática é o usuário e a promoção da saúde e fortalecimento da vida deste, e não a concepção metodológica do profissional em si e para si, esta apenas aponta caminhos para que seja possível alcançar a meta almejada. 


\section{REFERÊNCIAS}

Antunes, D. S. H. y (2011). Buscando Saúde e Plenitude: a experiência de um grupo de Práticas Integrativas e complementares no Sistema Único de Saúde. EFDeportes - Revista digital, 16(163)

Antunes, D. S. H. (2014). A criação da politica municipal de práticas integrativas e complementares no município do Rio Grande/RS: desafios, conquistas e perspectivas. Trabalho de conclusão do curso de Residência Multiprofissional em Saúde da Família. Universidade Federal do Rio Grande, Rio Grande.

Bagrichevsky M., Palma A., Estevão A. \& Da Ros M. (org.), (2007). A Saúde em Debate na Educação Física - Volume 2. Blumenau: editora da UFSC.

Bagrichevsky M., Palma A., Estevão A. \& Da Ros M. (org.), (2006). A Saúde em Debate na Educação Física - Volume 2. Blumenau: Nova Letra, 240p.

Bagrichevsky M., Palma A., Estevão A. \& Da Ros M. (org.), (2003). A Saúde em Debate na Educação Física - Volume 1. Blumenau: Edibes.

Baptista, T.J.R. (2013). Corpo, Estética, Exercício e Saúde Coletiva. Praxia - Revista on line de Educação Física da $U E G, 1$, p. 4-24.

Baptista, T.J.R. \& Resende, A.C.A. (2009). Educação do Corpo: produção e reprodução. Inter-ação (UFG. Online)34, p. 01-19.

Baptista, T.J.R. (2007). Educação do corpo: produção e reprodução. 2007. 152 f. Tese (Doutorado em Ciências Humanas) - Universidade Federal de Goiás, Goiânia.

Barollo, C. \& Cabral, M. (2012). Um dos maiores programa de práticas integrativas e complementares da América Latina: São Paulo. Área Técnica de Medicina Tradicional, Homeopatia e Práticas Integrativas em Saúde da Coordenação da Atenção Básica da Secretaria Municipal de Saúde\# São Paulo\#SP. Rev bras med fam comunidade. Florianópolis, Jun:7.

Brasil. (2006a). Ministério Da Saúde. Secretaria de Vigilância em Saúde. Secretaria de Atenção à Saúde. Política Nacional de Promoção da Saúde. 3a edição. Série B. Textos Básicos de Saúde. Série Pactos pela Saúde, v. 7. Recuperado em 08 dezembro, 2016, de http://bvsms.saude.gov.br/bvs/publicacoes/politica_nacional_promoc ao_saude_3ed.pdf.

Brasil (2006b). Ministério da Saúde. Secretaria de Atenção à Saúde. Departamento de Atenção Básica. Política Nacional de Práticas Integrativas e Complementares no SUS: atitude de ampliação de acesso. Brasília, DF.

Brasil (2014). Ministério da Saúde. Secretaria de Atenção à Saúde. Departamento de Atenção Básica. Coordenação Nacional de Práticas Integrativas e Complementares. RELATÓRIO DE GESTÃO 2006/2010: Práticas Integrativas e Complementares no SUS. Brasília- DF.

Brasília. (2012). Experiência em Práticas Integrativas da Secretaria de Saúde do Distrito Federal. Apresentação $1^{\circ}$ Seminário Internacional de Práticas Integrativas e Complementares. Brasília, DF. Disponível em: http://189.28. 128.100/dab/docs/eventos/seminario_pnpic/dia14_05/dra_soraya_coury_brasil.pdf Acesso em: 04 jun. 2012.

Campos, G. W. de S. (2000). Saúde pública e saúde coletiva: Campo e núcleo de saberes e práticas. Sociedade e Cultura, 3(1), 51-74

Caspersen, C. J., Powell, K. E. \& Christenson, G. M. (1985). Physical activity, exercise, and physical fitness: definitions and distinctions for health-related research. Public Health Rep, 100(2), 126-131.

Cesana, J. (2011). Práticas Corporais Alternativas e Educação Física: entre a formação e a intervenção. 2011. $194 \mathrm{f}$. Tese (Doutorado em Educação Física)-Faculdade de Educação Física, Universidade Estadual de Campinas, Campinas.

Conselho Nacional de Saúde (CNS) (1997). Resolução número 218. Regulamentação das profissões de Saúde. Recuperado em 04 de junho, 2016, de http://conselho.saude.gov.br/resolucoes/reso_97.htm.

Coldebella, A. O. C. (2002). Práticas Corporais Alternativas: um caminho para formação em educação fisica (Dissertação). Universidade Estadual Paulista, Instituto de Biociências, Rio Claro, São Paulo.

Coletivo de Autores. (1992) Metodologia do ensino de educação física. São Paulo: Cortez. 
Corrêa, L. Q., Valério, M. P., Teixeira, A. O., Guerreiro, L. F., Silveira, D.F., Machado, P. T., Xavier, B. E., Oliz, M., Antunes, D. S. H.y \& Knuth, A. G. (2014). A atuação da Educação Física nas Residências Multiprofissionais em Saúde. Ver. Bras. Promoç. Saúde, 27(3), 428-433.

Dalla Zen, A.M. (2010) A Crise de paradigmas e a ressignificação do conhecimento para o século XXI. Em Questão, $16(2), 49-63$.

Foucault, M. (2004). A ética do cuidado de si como prática da liberdade. In: Ditos y Escritos V-Ética, Sexualidade, Política. Rio de Janeiro: Forense Universitária.

Damico, J. G. S. \& Knuth A. G. (2015). O des(encontro) das práticas corporais e atividade física: Hibridizações e borramentos no campo da saúde. Movimento, 20(1), 329-350.

Fiocruz. (2015). Fundação Oswaldo Cruz. Tai Chi Being Tao, Práticas Integrativas Corporais de auto cuidado, na praça da harmonia universal e nos postos de saúde do distrito federal Ideia SUS, banco de práticas e soluções em saúde e ambiente. Recuperado em 24 de setembro, 2017, de http://www.ideiasus.fiocruz.br/portal/index.php/gestao-participativa/874-tai-chi-being-tao-praticas-integr ativas-corporais-de-auto-cuidado-na-praca-da-harmonia-universal-e-nos-postos-de-saude-do-distrito-federal.

Florindo, A. A. \& Hallal, P. C. (Orgs) (2011). Epidemiologia da atividade fisica. Rio de Janeiro: Ateneu.

Fraga, A. B. \& Wachs, F. (Org.). Educação física e Saúde coletiva. Politicas de formação e perspectivas de intervenção. Porto Alegre: Editora UFRGS, 2007.

Grof, S. (2000). Psicologia do Futuro: lições da pesquisa moderna da consciência. Trad. Jussara de Avellar Serpa. Revisão técnica: Kiu Eckstein. Niterói: Heresis.

Goswami, A. Reed, R. \& Goswami, M. (2008). O Universo autoconsciente: como a consciência cria o mundo material. Tradução: Ruy Jungmann. 2a ed. São Paulo: Aleph.

Knuth, A. G. \& Loch, M. R. (2014). “Saúde é o que interessa, o resto não tem pressa”? Um ensaio sobre educação física e saúde na escola. Rev Bras Ativ Fis Saúde, 429-440.

Kuhn, T. S. (1992). A estrutura das revoluçôes cientificas. Trad. Beatriz Vianna Boeira e Nelson Boeira. $3^{a}$ Ed. São Paulo: Perspectiva.

Loch, M. R. (2012). Atividade Física e Saúde nos Programas de Pós-Graduação no Brasil: breve análise a partir de tomas Kuhn. Rev Bras Ativ Fis e Saúde, 17(1), 46-51.

Luz, M. T. (2007). Educação física e saúde coletiva: papel estratégico da área e possibilidades quanto ao ensino na graduação e integração na rede de serviços públicos de saúde. In. FRAGA, AB; WACHS, F (Org.). Educação física e Saúde coletiva. Políticas de formação e perspectivas de intervenção. Porto Alegre: Editora UFRGS.

Mizuno, J. \& Monteiro, H. L. (2012). Atribuições do profissional da Educação Física no campo da Saúde. Ver. Bras. Med. Fam. Comunidade, 7(1), 30.

Matthiesen, S. Q. (1999). A Educação Física e as Práticas Corporais Alternativas: a Produção Científica do Curso de Graduação em Educação Física da Unesp - Rio Claro De 1987 a 1997. Motriz, 5(2).

Neves, R. L. R., Antunes, P. C., Baptista, T. J. R. y Assumpção, L. O. T. (2015). Educação Física na saúde pública: Revisão Sistemática. R. bras. Ci. e Mov, 23(2), 163-177.

Nicolescu, B. (2005). O Manifesto da Transdisciplinaridade. Trad. Lucia Pereira Souza. $3^{a}$ ed. São Paulo: Triom.

Frosi, T.O. (2010). Lutando a saúde e a transcendência: experiências integrativas no Curso de Extensão em Karate da UFRGS 2010. EF deportes, 15(146). Recuperado em 26 de outubro, 2016, de http://www.efdeportes.com/efd 146/lutando-a-saude-e-a-transcendencia-experiencias-em-karate.htm..

Frosi, T. O. \& Pozatti, M. L. (2011) Práticas corporais integrativas e saúde emocional. Revista Didática Sistêmica, 13(1), 76-92

Pozatti, M. L. (2007) Buscando a inteireza do Ser: Proposiçôes para o desenvolvimento sustentável da consciência humana. Porto Alegre: Gênese.

Radin, D. (2008). Mentes Interligadas: evidências cientificas da telepatia, da clarividência e de outros fenômenos psíquicos. São Paulo: Aleph. 
Ramires, V., Becker, L., Sadovsky, A., Zago, A., Bielemann, R., \& Guerra, P. (2014). Evolução da pesquisa epidemiológica em atividade física e comportamento sedentário no Brasil: atualização de uma revisão sistemática. Rev Bras Ativ Fis e Saúde, 19(5), 529-530.

Santos, C. (2009) Promoção da saúde através da prática de atividade física Lian Gong. Revista Saúde Social, 18(1)

Santos, M. C. y Tesser, C. D. (2012). Um método para a implantação e promoção de acesso às Práticas Integrativas e Complementares na Atenção Primária à Saúde. Ciência Saúde Coletiva, 17(11).

São Paulo. (2012). Práticas Integrativas. Experiência em Várzea Paulista. XXIV congresso de secretários municipais de saúde do estado de São Paulo. Abril de 2010. Disponível em: http://www.conasems.org.br/files/APRESENTA cao_CONGRESSOSP2010.pdf Acesso em: 04 de junho de 2012.

Sheldrake, R. (1993). O renascimento da natureza: o reflorescimento da ciência e de Deus. São Paulo: Cultrix.

Sousa, I. M. C., Bodstein, R. C. A., Tesser, C. D., Santos, F. A. S., Hortale, V. A. (2012). Práticas integrativas e complementares: oferta e produção de atendimentos no SUS e em municípios selecionados. Cad. Saúde Pública, $28(11)$.

Universidade Federal do Ceará (UFC) (2015). Curso de Educação Física da UFC comemora 20 anos de criação. Recuperado em 24 de setembro, 2017, de http://ufc.br/noticias/noticias-de-2013/4514-curso-de-educacao-fis ica-da-ufc-comemora-20-anos-de-criacao.

Universidade Federal de Alagoas (UFAL) (2015).. Reitor acompanha atividades do Ufal Verão 2015. Recuperado em 24 de setembro, 2017 de http://www.ufal.edu.br/noticias/2015/01/reitor-acompanha-atividades-do-ufal-vera o-2015.

Universidade Federal de Itajuba (UNIFEI) (2015). Universidade Federal de Itajuba. Disciplinas ministradas na Graduação a partir de 2012. Recuperado em 24 de setembro, 2017, de http://www.anterior.unifei.edu.br/doc entes? $\mathrm{p}=$ paulonunes.

\section{BY-NC-SA}

